\title{
IncRNA MALAT-1 Predicts Prognosis of Patients with Systemic Lupus Erythematosus
}

\section{IncRNA MALAT-1 sagt die Prognose der Patienten mit systemischem Lupus erythematodes voraus}

Authors

Aihong Zhou ${ }^{1}$, Kunyun Mao², Guoqiang Liư ${ }^{3}$, Jun Li ${ }^{4}$ Xia Lin²

Affiliations

1 Department of Rheumatology immunology, The Second Affiliated Hospital of Shandong First Medical University, Tai'an, China

2 Department of Nephrology and Rheumatology, Dongping County People Hospital of Tai'an, Dongping, China

3 Department of Emergency, The Central Hospital of Tai'an, Tai'an, China

4 Department of Emergency, Coal Taishan sanatorium of Shandong Province (Shandong Tai'an Coal Mine Hospital), Dongping, China

Key words

MALAT-1, IncRNA, systemic lupus erythematosus, overall survival

Schlüsselwörter

MALAT-1, IncRNA, systemischer Lupus erythematodes, Gesamtüberleben

online publiziert $\quad 03.08 .2021$

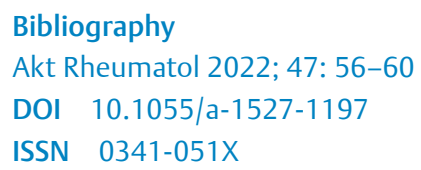

\section{ABSTRACT}

Objective To evaluate the association of MALAT- 1 and the prognosis of patients with systemic lupus erythematosus (SLE). Materials and Methods A total of 87 SLE patients and 50 healthy controls were recruited. The expression levels of MALAT- 1 were measured by quantitative PCR at baseline. Multivariate Cox regression analysis was performed to assess the association of MALAT-1 levels with survival after adjusting for potential confounding factors.

Results The MALAT- 1 levels in patients with SLE were significantly higher compared with healthy controls $(p<0.01)$. The 10 -year survival of patients with a higher MALAT- 1 level was significantly lower than that of patients with a lower MALAT-1 level $(\mathrm{HR}=5.54 ; 95 \% \mathrm{Cl}: 1.49-20.55 ; \mathrm{p}=0.01)$. Multivariate Cox regression indicated that the MALAT- 1 level was significantly associated with a risk of mortality $(\mathrm{HR}=10.02,95 \% \mathrm{Cl}: 1.14-$ $88.14, p=0.04$ ).

Conclusion The expression levels of MALAT- 1 may be a promising biomarker for evaluating the prognosis of SLE patients.

\section{ZUSAMMENFASSUNG}

Ziel Die Assoziation von MALAT-1 und der Prognose der Patienten mit systemischem Lupus erythematodes (SLE) zu bewerten. Material und Methoden Insgesamt wurden 87 SLE-Patienten und 50 gesunde Kontrollpersonen rekrutiert. Die Expressionsniveaus von MALAT-1 wurden zu Beginn der Studie durch quantitative PCR gemessen. Eine multivariate Cox-Regressionsanalyse wurde durchgeführt, um die Assoziation der MALAT-1-Spiegel mit dem Überleben nach Bereinigung um mögliche Störfaktoren zu bewerten.

Ergebnisse Die MALAT-1-Spiegel bei Patienten mit SLE waren im Vergleich zu gesunden Kontrollen signifikant höher $(P<0,01)$. Das 10-Jahres-Überleben von Patienten mit einem höheren MALAT-1-Spiegel war signifikant niedriger als bei Patienten mit einem niedrigeren MALAT-1-Spiegel ( $\mathrm{HR}=5,54$; $95 \% \mathrm{Cl}$ : 1,4920,55; $P=0,01$ ). Die multivariate Cox-Regression zeigte, dass der MALAT-1-Spiegel signifikant mit einem Mortalitätsrisiko assoziiert war ( $\mathrm{HR}=10,02,95 \% \mathrm{Cl}: 1,14-88,14, \mathrm{P}=0,04)$.

Schlussfolgerung Die Expressionsniveaus des MALAT-1-Niveaus könnten ein vielversprechender Biomarker für die Bewertung der Prognose von SLE-Patienten sein. 


\section{Introduction}

Systemic lupus erythematosus (SLE) is a severe autoimmune disease characterized by loss of immunological tolerance to autoantibodies to nuclear antigens, abnormal $\mathrm{T}$ - and $\mathrm{B}$-cell responses and autoantibody production [ 1,2$]$. It is a multisystem disease with heterogenous clinical manifestations including rash, arthritis, fatigue, nephritis, neurological disorders, anemia, and thrombocytopenia [3]. The prevalence of SLE is $20-50$ in every 100,000 people globally, while the number in China is as high as 70 per 100,000 people, which is significantly higher than Japan or other East Asia countries $[4,5]$. Both genetic and environment factors contribute to SLE pathogenesis, but the etiology of SLE is not fully understood [6].

Characterized as a subtype of noncoding RNAs with more than 200 nucleotides in length [7, 8], long noncoding RNAs (IncRNAs) are widely involved in various physiological and pathological processes mostly by functioning at transcriptional or posttranscriptional control [9]. Accumulating evidences showed that IncRNAs exist stably in human body fluids including urine and plasma, thereby acting as sensitive prognostic and diagnostic biomarkers in cancers, cardiovascular diseases, and immune diseases [10-12].

The metastasis-associated lung adenocarcinoma transcript 1 (MALAT-1) is a novel IncRNA, which is expressed from chromosome $11 q 13$ and encodes a gene of about $8.7 \mathrm{~kb}$ [13]. It has been linked to gene regulation and alternative splicing as well as cell proliferation, apoptosis, migration, and invasion [14]. It was abnormally upregulated and involved in metastasis of several cancers including lung cancer, prostate cancer, breast cancer, and hepatocelIular carcinoma [15]. In addition, MALAT-1 was reported to be correlated with autoimmune diseases such as myasthenia gravis [15] and multiple sclerosis [16]. A recent study indicated that MALAT- 1 was the key regulatory factor in the pathogenesis of SLE through regulating the expression of IL-21 and SIRT1 in monocytes of SLE patients [17]. However, the prognostic value of MALAT1 in SLE patients was unclear yet.

The present study aimed to evaluate the expression levels and the role of MALAT- 1 in predicting the prognostic value in patients with SLE.

\section{Methods}

\section{Patients}

A total of 87 SLE patients and 50 age- and sex- matched normal controls were recruited in the Central Hospital of Tai'an from Mar. 2009 to Dec. 2010. All of the enrolled patients fulfilled at least four of the 1997 update of American College of Rheumatology (ACR) revised criteria for SLE [18]. All included patients had not received any immune suppressant drugs, immune modulator or hormonal therapy. Those with malignant tumors, acute/chronic infection or other auto-immune disease were excluded. Disease activity was assessed for all patients using the Systemic Lupus Erythematosus Disease Activity Index (SLEDAI).

This study was approved by the ethic committee of our hospital and informed consent was obtained from all individual participants

\section{Data collection}

Demographic, clinical and laboratory data were obtained from first hospitalization medical records of SLE patients, e. g. age, sex, disease duration, SLEDAI, antinuclear antibody (ANA), white blood counts (WBCs), platelets, anti-double-stranded DNA antibody (anti-dsDNA $A b)$, anticardiolipin antibody (ACL), antinucleosome antibody (AnuA), anti-Sm antibody (Sm), anti-SSA antibody (SSA), 24-h proteinuria excretion, complement component 3 (C3), complement component 4 (C4) and organ involvement (mucocutaneous system, musculoskeletal system, cardiopulmonary system, nephropathy, blood system and neuropsychiatric system). Survival was determined from the time of SLE diagnosis to the last contact or date of death.

\section{mRNA expression of MALAT-1}

$5 \mathrm{ml}$ peripheral blood samples were collected from patients at admission and healthy controls during physical examination, then they were centrifuged at $3000 \mathrm{~g}$ for $10 \mathrm{~min}$. The supernatant serum was stored at $-80^{\circ} \mathrm{C}$ for up to three months. Total RNA was extracted from serum by miRNeasy extraction kit (Qiagen, Valenica, CA) using QIAzol lysis reagent. RNA was quantitated using NanoDrop2000 (Thermo scientific, USA). Reverse transcription was carried out on RNA in a final volume $20 \mathrm{ml}$ reactions using first strand Kit (Qiagen, Valenica, CA). Primers for RT-qPCR were MALAT-1: FGAATTGCGTCATTTAAAGCCTAGTT, R-GTTTCATCCTACCACTCCCAATTAAT; GAPDH: F-ACAGTCAGCCGCATCTTCTT, R-GACAAGCTTC CCGTTCTCAG. Quantitative mRNA expression was measured by ViiA ${ }^{\text {TM }} 7$ Real-Time PCR System (Applied Biosystems Inc., Foster City, CA, USA). The expression of GAPDH was detected as the endogenous control. Relative mRNA expression of MALAT-1 was calculated with the comparative threshold cycle $(\mathrm{Ct})\left(2^{-\Delta \Delta \mathrm{Ct}}\right)$ method.

\section{Statistical analysis}

Continuous variables are presented as median (quartiles) and compared with the Mann-Whitney U test. All categorical variables were summarized and expressed as proportions and compared with the use of chi-square test or Fisher's exact test, as appropriate. Cumulative survival curves were determined by the Kaplan-Meier method and event curves of disparate outcomes were compared by using the log-rank test. Cox regression analysis was used in death risk factors analysis, specifically presented as hazard ratio (HR) and $95 \%$ confidence intervals $(95 \% \mathrm{Cl})$. All tests were 2 -sided and a $P$ value of less than 0.05 was considered significant. All statistical analyses were performed with the SPSS statistical software program package (SPSS version 20.0 for Windows, Armonk, NY: IBM Corp.).

\section{Results}

The median age of 87 SLE patients was 37 years (range: $18-52$ years), which was comparable $(P=0.77)$ with 50 healthy controls (median 36 years, range: $18-52$ years). The gender distribution was also not significant between two groups $(P=0.55)$. The median disease duration of SLE patients was 40 months (10-77 months) and the median SLEDAI was 10 (5-15). The clinical and laboratory characteristics are presented in $>$ Table 1 . 
- Table 1 Baseline characteristics in patients with SLE and healthy controls.

\begin{tabular}{|c|c|c|c|}
\hline & $\begin{array}{c}\text { SLE } \\
\text { patients }\end{array}$ & $\begin{array}{l}\text { Healthy } \\
\text { controls }\end{array}$ & $\begin{array}{c}P \\
\text { Value }\end{array}$ \\
\hline \multicolumn{4}{|l|}{ Demographic variables } \\
\hline Age (years) & $37(18-52)$ & $36(18-52)$ & 0.77 \\
\hline Female, n (\%) & $80(92.0 \%)$ & $44(88.0 \%)$ & 0.55 \\
\hline Disease duration (months) & $40(10-77)$ & & \\
\hline SLEDAI & $10(5-15)$ & & \\
\hline \multicolumn{4}{|l|}{ Laboratory features } \\
\hline WBCs $\left(10^{3} / \mathrm{mm}^{3}\right)$ & $\begin{array}{c}4.3 \\
(1.9-8.9)\end{array}$ & & \\
\hline Platelets $\left(10^{3} / \mathrm{mm}^{3}\right)$ & $\begin{array}{c}263 \\
(29-389)\end{array}$ & & \\
\hline ANA positive, $\mathrm{n}(\%)$ & $81(93.1 \%)$ & & \\
\hline Anti-dsDNA Ab positive, $\mathrm{n}$ (\%) & $59(67.8 \%)$ & & \\
\hline ACL positive, $\mathrm{n}(\%)$ & $16(18.4 \%)$ & & \\
\hline AuaA positive, $\mathrm{n}$ (\%) & $31(35.6 \%)$ & & \\
\hline Sm positive, $\mathrm{n}(\%)$ & $26(29.9 \%)$ & & \\
\hline SSA positive, $n(\%)$ & $65(74.7 \%)$ & & \\
\hline $24 \mathrm{~h}$ proteinuria $>0.5 \mathrm{~g} / 24 \mathrm{~h}, \mathrm{n}(\%)$ & $47(54.0 \%)$ & & \\
\hline Decreased C3, n (\%) & $72(82.8 \%)$ & & \\
\hline Decreased C4, n (\%) & $48(55.2 \%)$ & & \\
\hline \multicolumn{4}{|l|}{ Organ involvement } \\
\hline Mucocutaneous system, n (\%) & $38(43.7 \%)$ & & \\
\hline Musculoskeletal system, n (\%) & $32(36.8 \%)$ & & \\
\hline Cardiopulmonary system, n (\%) & $14(16.1 \%)$ & & \\
\hline Nephropathy, n (\%) & $48(55.2 \%)$ & & \\
\hline Blood system, n (\%) & $29(33.3 \%)$ & & \\
\hline Neuropsychiatric system, n (\%) & $18(20.7 \%)$ & & \\
\hline
\end{tabular}

- Fig. 1 showed that the relative expression of MALAT-1 was significantly higher in SLE patients than that in healthy controls (SLE: median 1.93 , range $0.81-3.32$ vs. controls: median 0.92 , range $0.61-1.35 ; P<0.01)$. $>$ Table 2 presented that MALAT-1 level was significantly associated with WBCs $(r=0.50, P<0.01)$, platelets $(r=0.52, P<0.01)$ and nephropathy $(r=0.45, P<0.01)$.

The 5-year and 10-year survival rates for all SLE patients were $94.3 \%$ and $89.7 \%$, respectively. All SLE patients were divided into low MALAT-1 group or high MALAT-1 group according to the median value. The Kaplan-Meier curves and log-rank tests ( $\triangleright$ Fig. 2) showed that the high MALAT-1 level was associated with a higher risk of 10year mortality $(\mathrm{HR}=5.54$; $95 \% \mathrm{Cl}: 1.49-20.55 ; P=0.01)$.

Multivariate Cox proportional hazard regression is performed to explore the association of predictors with the risk of 10-year mortality in patients with SLE ( $\triangleright$ Table 3 ). It is shown that after adjusting potential confounding factors, a higher MALAT-1 level $(\mathrm{HR}=10.02,95 \% \mathrm{Cl}: 1.14-88.14, P=0.04)$, male $(\mathrm{HR}=17.96,95 \%$ Cl: 2.24-144.16, $P<0.01)$ and a higher SLEDAI level $(H R=13.43$,

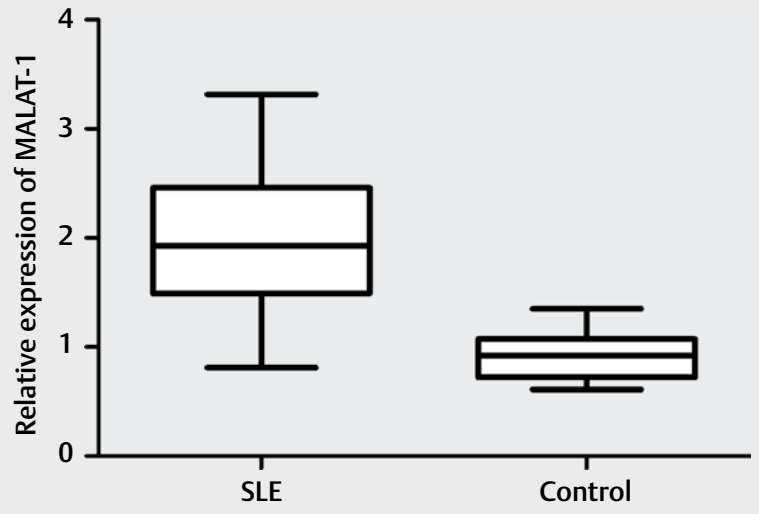

Fig. 1 Relative expression of MALAT-1 in SLE patients and healthy controls.

$95 \% \mathrm{Cl}: 1.53-117.92, P=0.02$ ) were all positively associated with the risk of 10 -year mortality.

\section{Discussion}

Despite great advances in modern medicines, the treatment of SLE remains unsatisfied, especially in its earlier stages. Therefore, to clearly elucidate the mechanism of SLE progression and to predict the disease course of SLE may allow better targeting of aggressive treatment to improve the therapeutic outcome [19]. The present study indicated that the mRNA expression of MALAT-1 was significantly upregulated in SLE patients compared with healthy controls. In addition, a higher MALAT- 1 level was associated with an increased risk of mortality in patients with SLE, after adjusting for potential confounding factors.

The 5-year and 10-year survival rates of 87 SLE patients included in this study were $94.3 \%$ and $89.7 \%$, similar to that in two large cohort studies in China and a global meta-analysis published recently [20-22]. Our results demonstrated that MALAT-1 level was positively associated with the risk of 10 -year mortality in patients with SLE. The mechanism of MALAT-1 as an independent risk factor affecting the prognosis of SLE is still unclear. In our study, there was a significant positive correlation between MALAT-1 level and both the WBC and platelet counts in the SLE patients. Platelet - leukocyte aggregation is essential for leukocyte recruitment, an important step of inflammatory and immune reactions and is partly regulated by MALAT-1 [23]. In addition, nephropathy were also significantly related with MALAT-1. Lupus nephritis (LN) is a major risk factor for morbidity and mortality in SLE and $10 \%$ of patients with LN will develop end stage renal disease (ESRD) [24]. In contrast with a non-significant correlation in our study, the correlation between MALAT-1 level and SLEDAI was reported to be significant in Fouad et al.'s study $(r=0.36, P=0.024)$ [25]. Disease activity was also demonstrated to be associated with mortality in patients with SLE [26].

In line with previous studies, male and high SLEDAI scores were shown to be associated with a higher risk of mortality [22, 27]. Pre- 
- Table 2 Correlation of MALAT-1 level with baseline characteristics in SLE patients.

\begin{tabular}{|l|r|r|}
\hline & Spearman's r & $\boldsymbol{P}$ Value \\
\hline Demographic variables & & \\
\hline Age & -0.18 & 0.10 \\
\hline Gender & -0.12 & 0.26 \\
\hline Disease duration & 0.02 & 0.83 \\
\hline SLEDAl & 0.09 & 0.41 \\
\hline Laboratory features & & \\
\hline WBCs & $\mathbf{0 . 5 0}$ & $<\mathbf{0 . 0 1}$ \\
\hline Platelets & $\mathbf{0 . 5 2}$ & $<\mathbf{0 . 0 1}$ \\
\hline ANA positive & 0.14 & 0.19 \\
\hline Anti-dsDNA Ab & -0.07 & 0.53 \\
\hline ACL & 0.03 & 0.77 \\
\hline AuaA & 0.04 & 0.71 \\
\hline Sm & -0.01 & 0.99 \\
\hline SSA positive & 0.08 & 0.47 \\
\hline 24h proteinuria $>0.5 \mathrm{~g} / 24 \mathrm{~h}$ & -0.18 & 0.09 \\
\hline Decreased C3 & 0.10 & 0.36 \\
\hline Decreased C4 & 0.21 & 0.05 \\
\hline Organ involvement & & 0.63 \\
\hline Mucocutaneous system & 0.05 & 0.70 \\
\hline Musculoskeletal system & 0.09 & \\
\hline Cardiopulmonary system & -0.06 & $\mathbf{0 . 4 5}$ \\
\hline Nephropathy & 0.05 & \\
\hline Blood system & 0.03 & \\
\hline Neuropsychiatric system & & \\
\hline & & \\
\hline
\end{tabular}

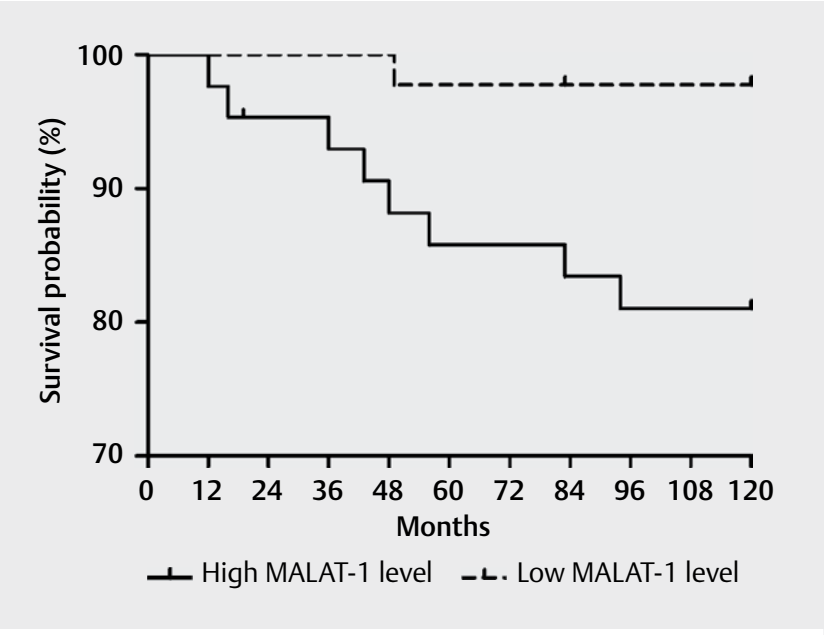

- Fig. 2 Kaplan-Meier survival curves by MALAT-1 level.

vious findings have implicated sexual disparities in the course of SLE, which may be caused by interactions between sex hormones, the immune system, genetic factors, and epigenetic modifications
- Table 3 Risk factors for mortality of patients with SLE using Multivariate Cox regression analysis.

\begin{tabular}{|l|r|r|}
\hline & \multicolumn{2}{|c|}{ Multivariate analysis } \\
\hline & HR (95\% CI) & P Value \\
\hline $\begin{array}{l}\text { High vs. low MATAL-1 } \\
\text { level }\end{array}$ & $10.02(1.14-88.14)$ & 0.04 \\
\hline $\begin{array}{l}\text { Male vs. Female } \\
\text { SLEDAI } \geq 10 \text { vs. }<10\end{array}$ & $17.96(2.24-144.16)$ & $<0.01$ \\
\hline
\end{tabular}

in influencing SLE progression [28]. SLEDAI score is the disease activity score of SLE, suggesting higher disease activity, more severe disease condition, higher treatment difficulty, and inevitably worse prognosis.

This study has some limitations. Firstly, the sample size was relatively small, a larger-scale longitudinal study with more patients should be required to validate the results. Second, only hospitalized patients with SLE were included in this study, which means that all the conclusions were conditional on having relatively serious SLE.

In conclusion, this study demonstrates for the first time that the expression of MALAT- 1 level has potential to be a prognostic indicator in patients with SLE.

\section{Conflict of Interest}

The authors declare that they have no competing interests.

\section{References}

[1] Grammatikos AP, Kyttaris VC, Kis-Toth K et al. A T cell gene expression panel for the diagnosis and monitoring of disease activity in patients with systemic lupus erythematosus. Clin Immunol 2014; 150: 192-200. doi:10.1016/j.clim.2013.12.002

[2] Kang SC, Hwang SJ, Chang YS et al. Characteristics of comorbidities and costs among patients who died from systemic lupus erythematosus in Taiwan. Arch Med Sci 2012; 8: 690-696. doi:10.5114/ aoms.2012.30293

[3] Fava A, Petri M. Systemic lupus erythematosus: Diagnosis and clinical management. J Autoimmun 2019; 96: 1-13. doi:10.1016/j. jaut.2018.11.001

[4] Osio-Salido E, Manapat-Reyes H. Epidemiology of systemic lupus erythematosus in Asia. Lupus 2010; 19: 1365-1373. doi:10.1177/0961203310374305

[5] Zou YF, Feng CC, Zhu JM et al. Prevalence of systemic lupus erythematosus and risk factors in rural areas of Anhui Province. Rheumatol Int 2014; 34: 347-356. doi:10.1007/s00296-013-2902-1

[6] Du Y, Sun F, Zhou M et al. The expression and clinical significance of different forms of LILRA3 in systemic lupus erythematosus. Clin Rheumatol 2019; 38: 3099-3107. doi:10.1007/s10067-019-04624-z 
[7] Kapranov P, Cheng J, Dike S et al. RNA maps reveal new RNA classes and a possible function for pervasive transcription. Science 2007; 316 : 1484-1488. doi:10.1126/science. 1138341

[8] Patel SA, DeMichele A. Adding Adjuvant Systemic Treatment after Neoadjuvant Therapy in Breast Cancer: Review of the Data. Curr Oncol Rep 2017; 19: 56. doi:10.1007/s11912-017-0613-6

[9] Wang KC, Chang HY. Molecular mechanisms of long noncoding RNAs. Mol Cell 2011; 43: 904-914. doi:10.1016/j.molcel.2011.08.018

[10] Spizzo R, Almeida MI, Colombatti A et al. Long non-coding RNAs and cancer: a new frontier of translational research? Oncogene 2012; 31 : 4577-4587. doi:10.1038/onc.2011.621

[11] Knauss JL, Sun T. Regulatory mechanisms of long noncoding RNAs in vertebrate central nervous system development and function. Neuroscience 2013; 235: 200-214. doi:10.1016/j.neuroscience.2013.01.022

[12] Schonrock N, Harvey RP, Mattick JS. Long noncoding RNAs in cardiac development and pathophysiology. Circ Res 2012; 111: 1349-1362. doi:10.1161/CIRCRESAHA.112.268953

[13] Ji P, Diederichs S, Wang W et al. MALAT-1, a novel noncoding RNA, and thymosin beta4 predict metastasis and survival in early-stage non-small cell lung cancer. Oncogene 2003; 22: 8031-8041. doi:10.1038/sj.onc. 1206928

[14] Eissmann M, Gutschner T, Hammerle M et al. Loss of the abundant nuclear non-coding RNA MALAT1 is compatible with life and development. RNA Biol 2012; 9: 1076-1087. doi:10.4161/rna.21089

[15] Kong X, Wang J, Cao Y et al. The long noncoding RNA MALAT-1 functions as a competing endogenous RNA to regulate MSL2 expression by sponging miR-338-3p in myasthenia gravis. J Cell Biochem 2019; 120: 5542-5550. doi:10.1002/jcb.27838

[16] Cardamone G, Paraboschi EM, Solda G et al. Not only cancer: the long non-coding RNA MALAT1 affects the repertoire of alternatively spliced transcripts and circular RNAs in multiple sclerosis. Hum Mol Genet 2019; 28: 1414-1428. doi:10.1093/hmg/ddy438

[17] Yang $H$, Liang $N$, Wang $M$ et al. Long noncoding RNA MALAT-1 is a novel inflammatory regulator in human systemic lupus erythematosus. Oncotarget 2017; 8: 77400-77406. doi:10.18632/oncotarget.20490

[18] Hochberg MC. Updating the American College of Rheumatology revised criteria for the classification of systemic lupus erythematosus. Arthritis Rheum 1997; 40: 1725. doi:10.1002/art.1780400928
[19] Gheita TA, Abdel Rehim DM, Kenawy SA et al. Clinical significance of matrix metalloproteinase-3 in systemic lupus erythematosus patients: a potential biomarker for disease activity and damage. Acta Reumatol Port 2015; 40: 145-149

[20] Wang Z, Li M, Wang Y et al. Long-term mortality and morbidity of patients with systemic lupus erythematosus: a single-center cohort study in China. Lupus 2018; 27: 864-869. doi:10.1177/0961203317751852

[21] Mak A, Cheung MW, Chiew HJ et al. Global trend of survival and damage of systemic lupus erythematosus: meta-analysis and meta-regression of observational studies from the 1950 s to 2000 s. Semin Arthritis Rheum 2012; 41: 830-839. doi:10.1016/j.semarthrit.2011.11.002

[22] Ge J, Jin Z, Feng X et al. Creatinine clearance rate predicts prognosis of patients with systemic lupus erythematosus: a large retrospective cohort study. Clin Rheumatol 2020, doi:10.1007/s10067-020-05485-7

[23] Masoumi F, Ghorbani S, Talebi F et al. Malat1 long noncoding RNA regulates inflammation and leukocyte differentiation in experimental autoimmune encephalomyelitis. J Neuroimmunol 2019; 328: 50-59. doi:10.1016/j.jneuroim.2018.11.013

[24] Alarcon GS. Multiethnic lupus cohorts: what have they taught us? Reumatol Clin 2011; 7: 3-6. doi:10.1016/j.reuma.2010.11.001

[25] Fouada NA, Shaker OG, Mohamed EA et al. Diagnostic potential of metastasis-associated-lung-adenocarcinoma-transcript-1 (MALAT-1) and TNF $\alpha$ and hnRNPL related immunoregulatory long non-coding RNA (THRIL) in systemic lupus erythematosus patients: Relation to disease activity. The Egyptian Rheumatologist 2019; 41: 197-201

[26] Fors Nieves CE, Izmirly PM. Mortality in Systemic Lupus Erythematosus: an Updated Review. Curr Rheumatol Rep 2016; 18: 21. doi:10.1007/s11926-016-0571-2

[27] Feng X, Pan W, Liu L et al. Prognosis for Hospitalized Patients with Systemic Lupus Erythematosus in China: 5-Year Update of the jiangsu Cohort. PLoS One 2016; 11: e0168619. doi:10.1371/journal. pone.0168619

[28] Tedeschi SK, Bermas B, Costenbader KH. Sexual disparities in the incidence and course of SLE and RA. Clin Immunol 2013; 149: 211-218. doi:10.1016/j.clim.2013.03.003 